\title{
KODAIRA DIMENSION OF SUBVARIETIES II
}

\author{
THOMAS PETERNELL
}

\section{Contents}

\begin{tabular}{|lr|}
\hline 1. Introduction & 1 \\
\hline 2. Preliminaries & 1 \\
\hline 3. A criterion for uniruledness & 2 \\
\hline 4. Computing invariants & 6 \\
\hline 5. A criterion for rational connectivity & 8 \\
\hline References & 10 \\
\hline
\end{tabular}

\section{INTRODUCTION}

This paper continues the study of non-general type subvarieties begun in [PSS99]. Consider for simplicity a submanifold $A$ of a projective manifold $X$ with ample normal bundle $N_{A}$ (but we will also consider also subvarieties with weaker positivity assumptions on the normal bundle). In [PSS99] it was shown that if $A$ is not of general type, then the Kodaira dimension $\kappa(X)=-\infty$. Conjecturally any manifold of negative Kodaira dimension is uniruled, i.e. covered by rational curves. Here we establish that in the above situation. The new ingredient is the paper [BDPP04] where it is shown that a manifold is uniruled if its canonical bundle $K_{X}$ is not pseudo-effective, i.e. its Chern class is not contained in the closure of the cone generated by effective classes. Alternatively, $K_{X}$ is not pseudo-effective, if $K_{X}$ does not admit a possibly singular metric with non-negative curvature (current).

Once $X$ is uniruled, one can form the rational quotient $f: X \rightarrow W$, see [Ca81],[Ko96]. This is just a rational map, but has some good properties, see (2.1). Now by a fundamental result of Graber-Harris-Starr [GHS03], $W$ is no longer uniruled, hence again by [BDPP04] $K_{W}$ is pseudo-effective ( $W$ can be taken smooth). Coming back to our submanifold $A$, this can be used to show that $f \mid A$ is onto $W$; thus we obtain a bound for $\operatorname{dim} W$ and often also for $\kappa(W)$. It allows also to relate $\pi_{1}(A)$ and $\pi_{1}(X)$. Finally we give a cohomological criterion for a projective manifold to be rationally connected.

For interesting discussions I would like to thank Frédéric Campana; I also thank the referee for important remarks. The paper was written within the framework of the priority program "Global Methods in Complex Geometry" of the Deutsche Forschungsgemeinschaft.

\section{Preliminaries}

(2.1). Let $X$ be a uniruled projective manifold. By identifying sufficiently general points which can be joined by chains of rational curves, one obtains the 
so-called rational quotient (e.g. [Ca81], [CP99], [Ko96])

$$
f: X \rightarrow W
$$

which has the remarkable property to be almost holomorphic, ie. the locus of indeterminacy does not project onto $W$. By [GHS03], $W$ does not carry any covering family of rational curves, ie. $W$ is not uniruled, and consequently the canonical bundle $K_{W}$ is not pseudo-effective (if $W$ is smooth, [BDPP04]).

If $\operatorname{dim} W=0$, then $X$ is rationally connected.

(2.2). In [Ca95], Campana introduced a refined Kodaira dimension, which potentially differs from the usual notion only in the case that $X$ is uniruled. Namely, he considers subsheaves $\mathcal{F} \subset \Omega_{X}^{p}$ of an arbitrary rank $r>0$ for any $p$ and defines

$$
\kappa^{+}(X)
$$

to be the maximum over all $\kappa(\operatorname{det} \mathcal{F})$, where $\operatorname{det} \mathcal{F}=\left(\bigwedge^{r} \mathcal{F}\right)^{* *}$. Conjecturally $\kappa^{+}(X)=\kappa(X)$ if $X$ is not uniruled; and $\kappa(X)=\kappa(W)$, when $X$ is uniruled with rational quotient $W$ - here we let $\kappa(W)=-\infty$ if $\operatorname{dim} W=0$. See [Ca95],[CP99] for details. In [CP04] it is proved that $\kappa^{+}(X)=\operatorname{dim} X$ implies $\kappa(X)=\operatorname{dim} X$.

(2.3) (1) We say that a vector bundle $E$ is $\mathbb{Q}$-effective [PSS99], if some symmetric power $S^{k}(E)$ is generically spanned by global sections.

(2) $E$ is almost ample, if $E$ is nef and $E \mid C$ is ample on every curve not contained in some countable union of proper subvarieties.

(3) $E$ is generically ample, if $E$ is $\mathbb{Q}$-effective and if $E \mid C$ is ample for all curves not contained in a countable union of proper subvarieties.

(4) $E$ is big if for all torsion free quotients $E \rightarrow \mathcal{S} \rightarrow 0$ of rank 1 the determinant $\operatorname{det} \mathcal{S}:=\mathcal{S}^{* *}$ is big.

As an example, consider a birational map $\pi: \hat{X} \rightarrow X$ from a projective manifold $\hat{X}$. Consider a vector bundle $E$ of the form $E=\pi_{*}(\hat{E})^{* *}$ with an ample vector bundle $\hat{E}$ on $\hat{X}$. Then $E$ is $\mathbb{Q}$-effective, big and generically ample.

Notice also that an almost ample vector bundle might not be big.

\section{A CRITERION FOR UNIRULEDNESS}

For the entire section we fix a projective manifold and a compact submanifold $A \subset X$ whose normal bundle $N_{A}=N_{A / X}$ is supposed to be $\mathbb{Q}$-effective. One could allow certain singularities (see remark 3.7(4)) but for sakes of simplicity we only consider the smooth case.

3.1. Lemma. Let $L$ be a pseudo-effective line bundle on $X$. Then the restriction $L_{A}$ is again pseudo-effective.

Proof. Fix an ample line bundle $H$ on $X$. Since $L$ is pseudo-effective, we find for any $m \gg 0$ a positive number $t$ such that

$$
H^{0}(X, t(m L+H)) \neq 0 .
$$

Then from [PSS99,2.1] we deduce the existence of some number $k$ such that

$$
H^{0}\left(S^{k} N_{A}^{*} \otimes t\left(m L_{A}+H_{A}\right)\right) \neq 0 .
$$

By assumption there exists $l$ such that $S^{l} N_{A}$ is generically spanned, hence $S^{l} N_{A}^{*} \subset$ $\mathcal{O}_{A}^{q}$. Putting things together, we obtain a number $t^{\prime}$ such that

$$
H^{0}\left(t^{\prime}\left(m L_{A}+H_{A}\right)\right) \neq 0 .
$$


Thus $L_{A}$ is pseudo-effective.

As application we prove

3.2. Theorem. If $N_{A}$ is $\mathbb{Q}$-effective and $\operatorname{det} N_{A}$ is big and $A$ not of general type, then $X$ is uniruled.

Proof. We are going to show that $K_{X}$ is not pseudo-effective; then the uniruledness follows from [BDPP04]. Assume to the contrary that $K_{X}$ is pseudo-effective. Then by the previous lemma $K_{X} \mid A$ is pseudo-effective. Thus by adjunction, $K_{A}$ is the sum of a pseudo-effective and a big divisor, hence big, contradicting our assumption.

3.3. Remark. In [PSS99] it is shown that if $N_{A}$ is $\mathbb{Q}$-effective and $\kappa\left(\operatorname{det} N_{A}\right)>$ $\kappa(A)$, then $\kappa(X)=-\infty$. So we would expect that in Theorem 3.2 that it is sufficient to assume $\kappa\left(\operatorname{det} N_{A}\right)>\kappa(A)$. ¿From our discussion in the proof we see that things come down to the following. Suppose that

$$
K_{A}=P+E
$$

with $P$ pseudo-effective and $E$ effective, then we should have $\kappa(A) \geq \kappa(E)$. This is discussed in [CP04] and proved in special cases. It is not unreasonable to expect that the assumption on the $\mathbb{Q}$-effectivity can be substituted by assuming that $N_{A}$ is nef.

3.4. Corollary. Let $X$ be a projective manifold, $A \subset X$ a submanifold with ample normal bundle. If $A$ is not of general type, then $X$ is uniruled.

3.5. Setup. Returning to submanifolds $A \subset X$ with $\mathbb{Q}$-effective normal bundle $N_{A}$ and big determinant $\operatorname{det} N_{A}$ and assuming again $A$ not of general type, we now know that $X$ is uniruled. So let

$$
f: X \rightarrow W
$$

be "the" rational quotient; we choose $W$ to be smooth. Since $W$ is not uniruled, $K_{W}$ is pseudo-effective and if $p=\operatorname{dim} W$, there exists a locally free pseudo-effective sheaf $\mathcal{F} \subset \Omega_{X}^{p}$ of rank 1. To be more precise, consider a birational map $\pi: \hat{X} \rightarrow X$ from a projective manifold $\hat{X}$ such that the induced map $\hat{f}: \hat{X} \rightarrow W$ is holomorphic. Then we have an injective map $\hat{f}^{*}\left(\Omega_{W}^{p}\right) \rightarrow \Omega_{\hat{X}}^{p}$ and we set

$$
\mathcal{F}=\left(\pi_{*}\left(\hat{f}^{*}\left(K_{W}\right)\right)\right)^{* *} .
$$

Then $\mathcal{F} \subset \Omega_{X}^{p}$ since this evidently holds outside a set of codimension at least 2 and $\mathcal{F}$ is clearly pseudo-effective. Moreover, if $A \subset X$ with $N_{A} \mathbb{Q}$-effective is a subvariety then Lemma 3.1 shows that $\mathcal{F}_{A}$ is again pseudo-effective.

If $A$ does not meet the set of indeterminacies of $f$, then the almost ampleness of $N_{A}$ forces $f \mid A$ to be onto as soon as $\operatorname{dim} A \geq \operatorname{dim} W$. This actually also holds if $A$ does meet the indeterminacy locus:

3.6. Lemma. Let $X$ and $W$ be projective manifolds, $f: X \rightarrow W$ be almost holomorphic. Let $A \subset X$ be a submanifold or a local complete intersection with almost ample normal bundle and assume $\operatorname{dim} A \geq \operatorname{dim} W$. Then $A$ meets the general fiber of $f$, in particular $A$ is not contained in the indeterminacy locus of $f$, and $f: A \rightarrow W$ is dominant. 
Proof. If $N_{A}$ is ample, this is a special case of [FL82], Theorem 1 or Corollary 1, appplied to a general fiber or a general hyperplane section of a general fiber. See also [Fu84,12.2.4].

The more general case that $N_{A}$ is almost ample can be done by an easy adaption of the proof of the ample case.

It is not clear whether the lemma is still true when $N_{A}$ is only $\mathbb{Q}$-effective (or generically ample).

In the next theorem we make use of the notations of (3.5).

3.7. Theorem. Assume that $A$ is not of general type with $N_{A}$ big and $\mathbb{Q}$-effective. Then:

1.) $\mathcal{F}_{A}$ is a (pseudo-effective) subsheaf of $\Omega_{A}^{p}$.

2.) $\operatorname{dim} A \geq \operatorname{dim} W$, and if $N_{A}$ is almost ample or if $A$ is not in the indeterminacy locus of $f$, then $f \mid A$ is onto $W$.

3.) If $A$ is uniruled with rational quotient $A \rightarrow B$, then even $\operatorname{dim} B \geq \operatorname{dim} W$. If $N_{A}$ is ample or if $A$ is not in the indeterminacy locus of $f$, then $f \mid A$ is onto with positive-dimensional fibers; more generally there is a surjective meromorphic map $B \rightarrow W$.

Proof. Recall that indeed $X$ is uniruled and consider the rational quotient $f: X \rightarrow \rightarrow$ $W$. First we show how to derive (2),(3) and (4) from (1).

(2) is obviously a consequence of (1); for the second part use Lemma 3.6 and observe that since $\mathcal{F}_{A}$ is a subsheaf of $\Omega_{A}^{p}$ via the canonical generically defined map $f^{*}\left(\Omega_{W}^{p}\right) \rightarrow \Omega_{A}^{p}$, the restricted map $f \mid A$ must be onto.

Concerning (3): if $A$ is uniruled, then we cannot have $\operatorname{dim} A=p$, because then $K_{A}$ would contain a pseudo-effective line bundle, i.e. would be pseudo-effective. More generally, consider a general smooth fiber $A_{b}$ of the almost holomorphic map $g: A \rightarrow B$. Then $\Omega_{A}^{p} \mid A_{b}$ has a filtration by terms

$$
\Omega_{A_{b}}^{i} \otimes \bigwedge^{j} N_{A_{b}}^{*} .
$$

Since $A_{b}$ is rationally connected, $\mathcal{F}_{A}$ cannot be in a term $\Omega_{A_{b}}^{i} \otimes \bigwedge^{j} N_{A_{b}}^{*}$ with $i>0$, hence we must have $\mathcal{F}_{A} \subset g^{*}\left(\Omega_{B}^{p}\right)$, at least generically. But then $\operatorname{dim} B \geq p$. If $A$ is not contained in the indeterminacy locus of $f$, then it is clear that the fibers $A_{b}$ must be contracted by $f$ (consider general rational curves in $A_{b}$; their deformations cover all of $X$ ). Hence $X \rightarrow W$ factors over $B \rightarrow W$.

It remains to prove (1). By taking $\wedge^{p}$ of the exact sequence

$$
0 \rightarrow N_{A}^{*} \rightarrow \Omega_{X}^{1} \mid A \rightarrow \Omega_{A}^{1} \rightarrow 0
$$

we obtain a filtration of $\Omega_{X}^{p} \mid A$ by the terms

$$
\Omega_{A}^{j} \otimes S^{i} N_{A}^{*}
$$

with $i+j=p$. The map $\mathcal{F} \rightarrow \Omega_{X}^{p}$ corresponds to a non-zero section $s \in H^{0}\left(X, \Omega_{X}^{p} \otimes\right.$ $\left.\mathcal{F}^{*}\right)$. If $s \mid A=0$, choose $k$ maximal such that

$$
s \in H^{0}\left(X, \Omega_{X}^{p} \otimes \mathcal{F}^{*} \otimes \mathcal{I}_{A}^{k}\right) .
$$

Allowing $k=0$ if $s \mid A \neq 0$, we always get a non-zero map

$$
S^{k} N_{A} \otimes \mathcal{F}_{A} \rightarrow \Omega_{X}^{p} \mid A .
$$


This yields a non-zero map

$$
S^{k} N_{A} \otimes \mathcal{F}_{A} \rightarrow \Omega_{A}^{j} \otimes S^{i} N_{A}^{*}
$$

and therefore a non-zero map

$$
S^{k} N_{A} \otimes S^{i} N_{A} \otimes \mathcal{F}_{A} \rightarrow \Omega_{A}^{j} .
$$

Since $\mathcal{F}_{A}$ is pseudo-effective (see 3.5) and since $N_{A}$ is assumed to be big, the determinant $\mathcal{L}$ of the image of this map is big. If now $i>0$ or $k>0$, then by [CP04], we obtain $\kappa(A)=\operatorname{dim} A$, contrary to our assumption. Hence $\mathcal{F}_{A} \subset \Omega_{A}^{p}$.

3.8. Corollary. Assume that $A$ is not of general type and $N_{A}$ to be $\mathbb{Q}$-effective. Then the conclusions of Theorem 3.7 hold, if one of the following conditions is satisfied.

1.) $N_{A}$ is ample.

2.) $\operatorname{det} N_{A}$ big; $A$ is not contained in the indeterminacy locus of the rational quotient; $W$ is of general type and $f \mid A$ is generically finite.

3.) $A$ is uniruled, $N_{A}$ is generically ample.

4.) $A$ is uniruled, $\kappa(W)>0$.

5.) For any map

$$
S^{k} N_{A} \otimes \mathcal{F}_{A} \rightarrow \Omega_{A}^{j}
$$

the determinant of the image is big.

Proof. (1) is clear. For (2) we only need to remark that $\mathcal{F}_{A}$ is big; then the proof of (3.7) still works. The bigness of $\mathcal{F}_{A}$ follows from the fact that $f \mid A: A \rightarrow W$ is generically finite, so that it suffices to show that $K_{W} \mid \overline{f(A)}$ is big. This in turn follows from the bigness of $K_{W}$, the fact that the normal sheaf of $\overline{f(A)} \subset W$ is $\mathbb{Q}$-effective and [PS99,4.2].

For (3) and (4), consider a general rational curve $C$ in $A$ and first observe that the deformations of $C$ fill up $X$ so that $X$ is uniruled. Then consider the restricted morphism

$$
\kappa_{C}: S^{k} N_{A}\left|C \otimes \mathcal{F}_{C} \rightarrow \Omega_{A}^{j}\right| C \otimes S^{i} N_{A} \mid C .
$$

Since $\mathcal{F}_{A}$ is pseduo-effective (3.5) and since $N_{A} \mid C$ is ample of $N_{A}$ is generically ample, $\kappa_{C}$ must vanish, the dual of $\Omega_{A}^{j} \mid C$ being nef. In case $N_{A}$ is just $\mathbb{Q}$-effective, we can only conclude that either $\kappa_{C}=0$ for general $C$ or does not have any zeroes. Hence if the full map $\kappa \neq 0$, then it can vanish only in codimension 2. Then consider a general curve $B$ so that $\kappa_{B}$ does not have zeroes. This is only possibly when $\mathcal{F}_{B}$ is not ample which implies $\mathcal{F} \equiv 0$, hence $\kappa(W)=0$.

(5) finally is a direct consequence from the proof of $(3.7)(1)$.

The critical point in the proof of Theorem 3.7 where we use the bigness of $N_{A}$ is the investigation of a line bundle $\mathcal{L} \subset \bigwedge^{r} \Omega_{A}^{j}$. If say $N_{A}$ is generically ample, then this line bundle has the property that

$$
L \cdot C>0
$$

for all movable curves $C$. Recall that a curve is movable, if the deformations of $C$ fill up the whole variety. In general, it is not true that a line bundle with this property is big. However the following should be true. 
3.9. Conjecture. Let $A$ be a projective manifold, $\mathcal{L} \subset \wedge^{r} \Omega_{A}^{j}$ a line bundle such that $\mathcal{L} \cdot C>0$ for all movable curves. Then $\mathcal{L}$ is big, in particular $A$ is of general type.

Even if $L=K_{A}$, this conjecture is unknown in dimension at least 4. However:

3.10. Proposition. Assume that $A$ admits a good minimal model $A^{\prime}$, i.e., some multiple $m K_{A^{\prime}}$ is spanned. Then Conjecture 3.9 holds in the weak form that $A$ is of general type.

Proof. It is easily checked that the line bundle $\mathcal{L}$ induces a Weil divisor $\mathcal{L}^{\prime}$ on $A$ which is $\mathbb{Q}$-Cartier such that

$$
\mathcal{L}^{\prime} \subset \bigwedge^{r} \Omega_{A^{\prime}}^{j}
$$

This last sheaf is by definition the reflexive extension of the corresponding sheaf on the smooth part. Notice that the existence of $\mathcal{L}$ forces $A$ to be non-uniruled. Consider the exact sequence

$$
0 \rightarrow \mathcal{L}^{\prime} \rightarrow \bigwedge^{r} \Omega_{A^{\prime}}^{j} \rightarrow Q \rightarrow 0 .
$$

Then $\operatorname{det} Q$ is generically nef by $[\mathrm{CP} 04,1.5]$. Taking determinants, we obtain

$$
N K_{A^{\prime}}=\mathcal{L}^{\prime}+\operatorname{det} Q
$$

for a suitable positive number $N$. Thus $K_{A^{\prime}} \cdot C>0$ for all movable curves. Since some multiple of $K_{A^{\prime}}$ is spanned, $A$ must be of general type.

3.11. Corollary. Assume $A \subset X$ not of general type and $\operatorname{dim} A \leq 3$. If $N_{A}$ is $\mathbb{Q}$-effective and generically ample with $\operatorname{det} N_{A}$ big, or, then Theorem 3.7 holds.

3.12. Remark. (1) The proof of (3.7) shows that $\kappa(W) \leq \kappa^{+}(A)$ (with the assumption that $A$ is not in the indeterminacy locus of $f$ in case $N_{A}$ is not almost ample).

(2) Via the conjectural equality $\kappa^{+}(X)=\kappa(W)$, one would arrive at

$$
\kappa^{+}(X) \leq \min \left(\operatorname{dim} A, \kappa^{+}(A)\right) .
$$

(3) There should be a version of (3.7) in the case $\kappa\left(\operatorname{det} N_{A}\right)>\kappa(A)$.

(4) Everything in this section works also if $X$ and $A$ have canonical singularities and $\operatorname{codim}_{A}(A \cap \operatorname{Sing}(X)) \geq 2$.

3.13. Example. Let $X$ be a projective manifold and $C \subset X$ an elliptic curve with ample normal bundle. Then $\operatorname{dim} W \leq 1$, i.e. $\kappa^{+}(X) \leq 0$. If $X$ is not rationally connected, i.e. $\operatorname{dim} W=1$, then the rational quotient $X \rightarrow W$ is holomorphic and $C$ is multi-section. In particular $W$ is elliptic and $q(X)=1$.

We will generalize that in the following section.

\section{Computing invariants}

In this section we compare the fundamental group and the spaces of $p$-forms for $A$ and $X$, when the normal bundle $N_{A}$ is almost ample and $\mathbb{Q}$-effective. If $N_{A}$ is ample, a theorem of Napier-Ramachandran [NR98] says that, given a submanifold $A \subset X$ with ample normal bundle, then the image of $\pi_{1}(A) \subset \pi_{1}(X)$ has finite index (instead of ampleness it actually suffices to assume finiteness of formal cohomology in degree 0 along $A$ of locally free sheaves; but the relation of this property to almost 
ampleness is unclear). We find Napier-Ramachandran's theorem again if $A$ is not of general type, but we can also weaken the ampleness assumption. Furthermore we can deal with holomorphic forms of any degree.

4.1. Theorem. Let $X$ be a projective manifold, $A \subset X$ a submanifold not of general type. Suppose that $N_{A}$ is big, $\mathbb{Q}$-effective and almost ample (or that $N_{A}$ is $\mathbb{Q}$-effective, almost ample and one of the conditions of (3.8) or (3.11) holds). Then

1.) The image of the canonical map

$$
\pi_{1}(A) \rightarrow \pi_{1}(X)
$$

has finite index in $\pi_{1}(X)$; the index is at most the number of connected components of the general fiber of $A$ over the rational quotient $W$.

2.) $h^{0}\left(\Omega_{X}^{q}\right) \leq h^{0}\left(\Omega_{A}^{q}\right)$ for all $q \geq 1$.

Proof. (1) We are using the followig basic fact: if $f: X \rightarrow Y$ is a surjective holomorphic map between normal compact complex spaces, then the image of $f_{*}: \pi_{1}(X) \rightarrow \pi_{1}(Y)$ has finite index in $\pi_{1}(Y)$ [Ca91,1.3], where the index is also computed. Since $X$ is smooth, then the same still holds for meromorphic dominant $f$ (blow up $X$ to make $f$ holomorphic; this does not change $\pi_{1}$ ).

We apply this to the rational quotient $f: X \rightarrow W$ to conclude via 3.6 and 3.7 that the image of

$$
\pi_{1}(A) \rightarrow \pi_{1}(W)
$$

has finite index. Now the claim follows from the isomorphism

$$
\pi_{1}(X) \simeq \pi_{1}(W)
$$

(Kollár [Ko93,5.2]).

(2) This follows immediately by considering a holomorphic model of $f \mid A$ and by observing $h^{0}\left(\Omega_{X}^{q}\right)=h^{0}\left(\Omega_{W}^{q}\right)$, the fibers of $f$ being rationally connected.

We are now applying (4.1) to special cases.

4.2. Corollary. Let $A \subset X$ be an abelian variety embedded in a projective manifold $X$. Suppose that $N_{A}$ is almost ample, $\mathbb{Q}$-effective and big.

1.) $q(X) \leq \operatorname{dim} A$ and the Albanese map is surjective.

2.) The image of

$$
\pi_{1}(A) \rightarrow \pi_{1}(X)
$$

has finite index in $\pi_{1}(X)$ and therefore $\pi_{1}(X)$ is almost abelian.

Proof. (1) is a direct consequence of the almost ampleness of $N_{A}$ while (2) is clear from (4.1).

4.3. Corollary. If $A \subset X$ is a simply connected submanifold not of general type with almost ample, $\mathbb{Q}$-effective and big normal bundle, then $\pi_{1}(X)$ is finite.

4.4. Remark. In (4.1) - (4.3) the assumption on almost ampleness is only used to make sure that $A$ is not contained in the indeterminacy locus of the rational quotient. Thus we can omit "almost ampleness" by requiring that $A$ is not contained in the indeterminacy locus. 
If $A \subset X$ is a submanifold whose normal bundle is almost ample without assumption on the Kodaira dimension or, more generally, generically ample (possible even with the generic spannedness assumption replaced by a generic nefness assumption), then one still would expect that the image of $\pi_{1}(A) \rightarrow \pi_{1}(X)$ has finite index. Here is a partial result in terms of the Shafarevitch map [Ko93],[Ca95]

$$
\text { sh }: X \rightarrow \operatorname{Sh}(X),
$$

which is almost holomorphic.

4.5. Proposition. Let $X$ be a projective manifold. Let $A \subset X$ be a submanifold or a local complete intersection. Suppose that

(1) $N_{A}$ is almost ample, or that

(2) A contains a local complete intersection $B$ such that $N_{B / X}$ is ample.

If $\operatorname{dim} A \geq \operatorname{dim} S h(X)$, resp. $\operatorname{dim} B \geq \operatorname{dim} S h(X)$, then the image of $\pi_{1}(A) \rightarrow$ $\pi_{1}(X)$ has finite index.

Proof. We use the following fact which was communicated to me by F.Campana:

$\left.{ }^{*}\right)$ If $s h \mid A$ dominates $\operatorname{Sh}(X)$, then the image of $\pi_{1}(A)$ in $\pi_{1}(X)$ has finite index in $\pi_{1}(X)$.

To prove $\left(^{*}\right)$, let $h: \tilde{X} \rightarrow X$ be the universal cover and $\tilde{A}=h^{-1}(A)$. It suffices to show that $\tilde{A}$ has only finitely many irreducible components. Consider a general fiber $F$ of $s h$ and let $\tilde{F}=h^{-1}(F)$. Then $\tilde{F}$ is compact. Now every irreducible component $\tilde{A}_{i}$ of $\tilde{A}$ dominates $A$, hence $\tilde{A}_{i}$ meets $F$. Since $\tilde{A} \cap \tilde{F}$ has only finitely many components, we conclude $(*)$.

Now an application of (3.7) yields (1).

(2) By (3.7) $s h \mid B$ dominates Sh(X), hence $s h \mid A$ dominates $\mathrm{Sh}(\mathrm{X})$, and we can apply $(*)$.

Of course, this gives nothing when $\operatorname{dim} X=\operatorname{dim} S h(X)$, i.e. when there is no compact subvariety of positive dimension through the very general point of the universal cover $\tilde{X}$. In that case $X$ is of general type or $\chi\left(\mathcal{O}_{X}\right)=0$ [CP04].

\section{A CRITERion For RATIONAL CONNECTIVITY}

The following well-known conjecture is due to Mumford.

5.1. Conjecture. Let $X$ be a projective manifold. Assume that

$$
H^{0}\left(X,\left(\Omega_{X}^{1}\right)^{\otimes m}\right)=0
$$

for all $m \in \mathbb{N}$. Then $X$ is rationally connected.

This is slightly weaker than the conjecture that $\kappa^{+}(X)=-\infty$ implies rational connectedness (see (2.2)). In fact, $H^{0}\left(X,\left(\Omega_{X}^{1}\right)^{\otimes m}\right)=0$ implies that

$$
H^{0}\left(X, \Gamma\left(\Omega_{X}^{1}\right)\right)=0
$$

for all tensor representations $\Gamma$ and in particular $\kappa^{+}(X)=-\infty$. In this direction we prove

5.2. Theorem. Let $X$ be a projective manifold and $L$ be a big line bundle on $X$. If

$$
H^{0}\left(X,\left(\left(\Omega_{X}^{1}\right)^{\otimes m} \otimes L\right)^{\otimes N}\right)=0
$$

for all $m, N \gg 0$, then $X$ is rationally connected. 
Proof. The condition $\left(^{*}\right)$ implies in particular that

$$
H^{0}\left(N\left(m K_{X}+L\right)\right)=0
$$

for $m, N \gg 0$. Hence $K_{X}$ is not pseudo-effective, because otherwise $m K_{X}+L$ would be big. Therefore $X$ is uniruled by [BDPP04]. Let $f: X \rightarrow W$ be the rational quotient ( $W$ smooth) and suppose $\operatorname{dim} W>0$. By eventually blowing up $X$, we clearly may assume that $f$ is holomorphic. Choose a big line bundle $L^{\prime}$ on $W$ and choose $k$ so large that $k L-f^{*}\left(L^{\prime}\right)$ is still big. By substituting $L^{\prime}$ by a multiple and $k$ by a multiple, we may assume that $k L-f^{*}\left(L^{\prime}\right)$ has a section so that

$$
k L=f^{*}\left(L^{\prime}\right)+A
$$

with $A$ effective. By $(*)$ we also have

$$
H^{0}\left(X,\left(\left(\Omega_{X}^{1}\right)^{\otimes m} \otimes k L\right)^{\otimes N}\right)=0
$$

for large $m, N$. Therefore

$$
H^{0}\left(W,\left(\left(\Omega_{W}^{1}\right)^{\otimes m} \otimes L^{\prime}\right)^{\otimes N}\right) \subset H^{0}\left(\left(\left(\Omega_{X}^{1}\right)^{\otimes m} \otimes k L\right)^{\otimes N}\right)=0
$$

for $m, N \gg 0$ Hence by induction $W$ is rationally connected, contradiction. Actually it is sufficient to notice that

$$
H^{0}\left(N\left(m K_{W}+k L^{\prime}\right)\right)=0
$$

which shows that $K_{W}$ is not pseudo-effective, hence $W$ is uniruled, contradiction.

Of course, if $X$ is rationally connected, then

$$
H^{0}\left(X,\left(\left(\Omega_{X}^{1}\right)^{\otimes m} \otimes L\right)^{\otimes N}\right)=0
$$

for $m \gg 0$ and $N \in \mathbb{N}$. Just restrict to rational curves on which $T_{X}$ is ample.

5.3. Remark. By the same method one shows easily the following. If for every $p$ and every $\mathcal{F} \subset \Omega_{X}^{p}$ one has

$$
H^{0}(N(m \operatorname{det} \mathcal{F}+L))=0
$$

for some fixed big line bundle and every $m, N$, i.e. if $\operatorname{det} \mathcal{F}$ is not pseudo-effective, then $X$ is rationally connected.

5.4. Corollary. Let $X$ be a projective manifold, $C \subset X$ a (possibly singular) curve. If $T_{X} \mid C$ is ample, then $X$ is rationally connected.

Proof. We are going to verify $(*)$ in (5.2). Fix a big line bundle $L$ and choose $m_{0}$ so large that

$$
\left(\Omega_{X}^{1}\right)^{\otimes m_{0}} \otimes L \mid C
$$

is negative. Set

$$
\mathcal{E}=\left(\left(\Omega_{X}^{1}\right)^{\otimes m} \otimes L\right)^{\otimes N}
$$

with $m \geq m_{0}$ and $N \in \mathbb{N}$. Let $\hat{C}$ be the formal completion of $X$ along $C$. Then

$$
H^{0}(\mathcal{E}) \subset H^{0}(\mathcal{E} \mid \hat{C}) \subset \bigoplus_{k \geq 0} H^{0}\left(\mathcal{E} \otimes \mathcal{I}^{k} / \mathcal{I}^{k+1}\right) .
$$

Here $\mathcal{I}$ denotes the ideal sheaf of $C$. Since $\Omega_{X}^{1} \mid C$ is negative, the sheaves $\left(\mathcal{I}^{k} / \mathcal{I}^{k+1}\right) /$ torsion are negative; in fact, the canonical map

$$
\mathcal{I}^{k} / \mathcal{I}^{k+1} \rightarrow S^{k}\left(\Omega_{X}^{1} \mid C\right)
$$


is generically injective.

Hence any section in

$$
H^{0}\left(C, \mathcal{I}^{k} / \mathcal{I}^{k+1} \otimes \mathcal{E}\right)
$$

is a torsion section, supported at most in the singular locus of $C$. Thus if we take $s \in H^{0}(\mathcal{E})$, then the restriction to the $k-t h$ infinitesimal neighborhood is generically 0 . Since this holds for all $k$, we conclude that $s=0$. This shows $(*)$.

Similarly we prove:

5.5. Proposition. Let $X$ be a projective manifold and suppose that $T_{X} \mid C$ is nef for some (possibly singular) curve $C$.

(1) If $-K_{X} \cdot C>0$, then $X$ is uniruled;

(2) if $-K_{X} \cdot C=0$, then $\kappa(X) \leq \operatorname{dim} X-1$.

Proof. We adapt the proof of (5.4) and substitute $\Omega_{X}^{1}$ by $K_{X}$. We obtain for all $m$ and all line bundles $L$ :

$$
h^{0}\left(m K_{X}-L\right) \leq h^{0}\left(m K_{X}-L \mid \hat{C}\right) \leq \sum_{k} h^{0}\left(m K_{X}-L \otimes\left(\mathcal{I}^{k} / \mathcal{I}^{k+1}\right) / \text { tor }\right) .
$$

For $L=\mathcal{O}$ we obtain (1), and by plugging in some ample $L$, we deduce that $h^{0}\left(m K_{X}-L\right)=0$ for all $m$ so that $K_{X}$ cannot be big.

5.6. Remark. Assume that

$$
H^{0}\left(\left(\Omega_{X}^{1}\right)^{\otimes m}\right)=0
$$

for all positive $m$. Suppose that $K_{X}$ is pseudo-effective. If $K_{X}$ carries a metric with algebraic singularities [DPS01,2.14], then either $\chi\left(\mathcal{O}_{X}\right)=0$ - which is excluded by $(*)$ - or

$$
H^{0}\left(X, \Omega_{X}^{q} \otimes m K_{X}\right) \neq 0
$$

for some $q$ and infinitely many $m$. This also contradicts $(*)$. Consequently if $(*)$ holds, then $K_{X}$ cannot have a metric with algebraic singularities of non-negative curvature. In general, without the assumption on the metric (but still assuming $K_{X}$ to be pseudo-effective, of course), one can only conclude that

$$
\chi\left(X, \mathcal{O}\left((m+1) K_{X} \otimes \mathcal{I}\left(h_{m}\right)\right)=0\right.
$$

for all $m \geq m_{0}$ and all (singular) metrics $h_{m}$ on $m K_{X}$ with non-negative curvature (current). Actually all cohomology groups vanish:

$$
H^{0}\left(X, \mathcal{O}\left((m+1) K_{X}\right) \otimes \mathcal{I}\left(h_{m}\right)\right)=0 .
$$

\section{REFERENCES}

[BDPP04] Boucksom,S.;Demailly,J.P.;Paun,M.;Peternell,Th.: The pseudo-effective cone of a compact Kähler manifold and varieties of negative Kodaira dimension. math.AG 0405285

[Ca81] Campana,F.: Coréduction algébrique d'un espace analytique faiblement kählerien compact. Inv. math. 63, 187-223 (1981)

[Ca91] Campana,F.: On twistor spaces of the class $\mathcal{C}$. J. Differ. Geom. 33, 541-549 (1991)

[Ca95] Campana,F.: Fundamental group and positivity properties of cotangent bundles of compact Kähler manifolds. J. Alg. Geom. 4, 487-502 (1995)

[CP99] Campana,F.;Peternell,Th.: Recent developments in the classification theory of compact Kähler manifolds. Several Complex Variables, ed. M.Schneider, Y.T.Siu, MSRI Publications vol. 37, 113-159. Cambridge Univ. Press 1999

[CP04] Campana,F.;Peternell,Th.: Geometric stability of the cotangent bundle and the universal cover of a projective manifold. math.AG 0405093 to appear in Bull. Soc. Math. France 
[DPS01] Demailly,J.P.;Peternell,Th.;Schneider,M.: Pseudo-effective line bundles on compact Kähler manifolds. Intl. J.Math. 12, 689-741 (2001)

[FL82] Fulton,W.;Lazarsfeld,R.: Positivity and excess intersection. Enumerative Geometry and classical algebraic geometry; ed. P.Le Barz, Y.Hervier. Progress in Math. 24, 97-105, Birkhäuser (1982)

[Fu84] Fulton,W.: Intersection theory. Springer 1984

[GHS03] Graber,T.;Harris,J,;Starr,J.: Families of rationally connected varieties. J. Amer. Math. Soc. 16 (2003), no. 1, 57-67

[Ko93] Kollár,J.: Shafarevich maps and plurigenera of algebraic varieties. Invent. Math. 113, $177-215(1993)$

[Ko96] Kollár,J.: Rational curves on algebraic varieties. Erg. d. Math. 3.Folge, Band 32. Springer 1996

[NR98] Napier,T.;Ramachandran,M.: The $L^{2} \partial$-method, weak Lefschetz theorems, and the topology of Kähler manifolds. J. AMS 11, 375-396 (1998)

[PSS99] Peternell,Th.;Schneider,M.;Sommese,A.J.: Kodaira dimension of subvarieties. Int. J. Math. 10, 1065-1079 (1999)

Thomas Peternell

Mathematisches Institut

Universität Bayreuth

D-95440 Bayreuth, Germany

thomas.peternell@uni-bayreuth.de

FAX: + 921-552785 